07

\title{
Выявление очага разрушения методом рекуррентных графиков
}

\author{
() В.Л. Гиляров \\ Физико-технический институт им. А.Ф. Иофффе РАН, \\ Санкт-Петербург, Россия \\ Email: Vladimir.Hilarov@mail.ioffe.ru
}

(Поступила в Редакцию 1 июня 2017 г.)

Проанализированы рекуррентные графики (RP) и их числовые характеристики (RQA) для нормальной компоненты вектора смещения при возбуждении дефектной стальной плиты звуковым импульсом. Рассмотрены различные случаи распределения дефектов в пространстве - равномерное и нормальное, и выявлено различие RQA параметров в этих случаях.

Работа выполнена при финансовой поддержке Российского фонда фундаментальных исследований (проект № $16-05-00237 \mathrm{~A})$.

DOI: $10.21883 /$ FTT.2017.12.45234.180

Своевременное обнаружение роста очага разрушения является важной проблемой физики прочности материалов, поскольку ее решение может позволить предотвращать природные и техногенные катастрофы. Однако это решение осложняется тем, что разрушение материалов представляет собой нелинейный нестационарный процесс, и обычные методы, используемые в дефектоскопии, могут оказаться неэффективными при мониторинге действующих машин и конструкций. В настоящей работе предлагается применять для выявления растущего очага метод рекуррентных графиков.

Метод рекуррентных графиков основан на анализе близости точек траектории системы в фазовом пространстве, определяемой соотношением [1]

$$
\mathbf{R}_{i, j}(r, m)=\theta\left(\varepsilon_{i}-\left\|\mathbf{X}_{i}(m)-\mathbf{X}_{j}(m)\right\|\right),
$$

где $\mathbf{X}_{i}$ и $\mathbf{X}_{j}$ - фазовые траектории в $m$-мерном фазовом пространстве, $\theta-$ функция Хевисайда, a $\|\|-$, норма вектора. Рекуррентный график (RP) представляет собой двумерное представление матрицы (1), состоящее из нулей и единиц, причем близкие (рекуррентные) точки принято отображать черным цветом, а далекие белым. Близость точек определяется параметром $\varepsilon_{i}$. Способ выбора параметра $\varepsilon_{i}$ весьма важен для анализа конечного результата. Этот способ обсуждается в целом ряде работ (например, [2,3]), поэтому здесь на нем подробно останавливаться нет смысла. Заметим лишь, что в настоящей работе использовалось фиксированное число ближайших соседей (FAN [3]), подбираемое таким образом, чтобы плотность рекуррентных точек составляла примерно $1 \%$.

Для изучения совместного временного поведения двух различных динамических систем используют кроссрекуррентные графики, определяемые по аналогии с соотношением (1)

$$
C R_{i, j}(r, m)=\theta\left(\varepsilon_{i}-\left\|\mathbf{X}_{i}(m)-\mathbf{Y}_{j}(n)\right\|\right) .
$$

Здесь фазовые траектории $\mathbf{X}$ и $\mathbf{Y}$ относятся к разным динамическим системам. В этом случае возника- ет проблема сведения фазовых траекторий к одному масштабу, поскольку характерные масштабы изменения амплитуд двух систем могут быть различны. Однако использование метода FAN, фиксирующего одинаковую плотность рекуррентных точек автоматически решает эту проблему [2].

В работе [4] было предложено использовать метод рекуррентных графиков для изучения поврежденности материалов. В качестве модели материала с дефектами в [4] выступала стальная плита с боковыми надрезами разной длины. В статье [5] изучена стальная плита со случайным равномерным распределением пор в пространстве. В настоящей работе в качестве такого материала рассматривается плита со случайным нормальным распределением пор (модель очага разрушения). Полученные результаты сравниваются с результатами, полученными для равномерного распределения.

Объект исследования представляет собой прямоугольную стальную пластину размером $2.4 \times 1.6 \mathrm{~m}$ и толщиной $0.01 \mathrm{~m}$. Рассматривается плоская нестационарная задача теории упругости. На двух длинных торцах установлены точечные звуковые датчики, один из которых считается излучающим (с координатами $0,-0.8$ ), a три других на противоположном торце приемными (рис. $1, a)$. Координаты приемных датчиков: $(-1,0.8)$, $(0,0.8),(0.6,0.8)$. На излучающий датчик подается прямоугольный импульс силы шириной $1 \mathrm{~ms}$, сглаженный за время $10^{-5} \mathrm{~s}$, в направлении вертикальной оси $(y)$. На приемных датчиках записывается временная зависимость нормального смещения $\left(u_{y}\right)$ во временном интервале $0-0.01 \mathrm{~s}$, порожденного этим импульсом. Боковые концы пластины фиксировались (вектор смещения задавался равным нулю). Предполагалось, что деформации настолько малы, что материал остается линейным.

Внутри пластины делались круглые вырезы (поры). Координаты пор задавались генераторами псевдослучайных чисел с различными пространственными распределениями. Использовалось два типа распределения 


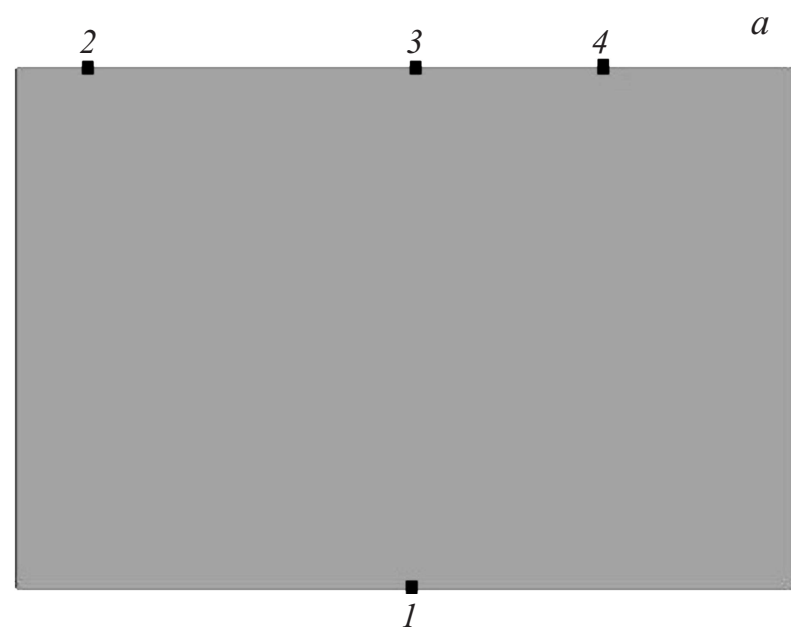

$b$
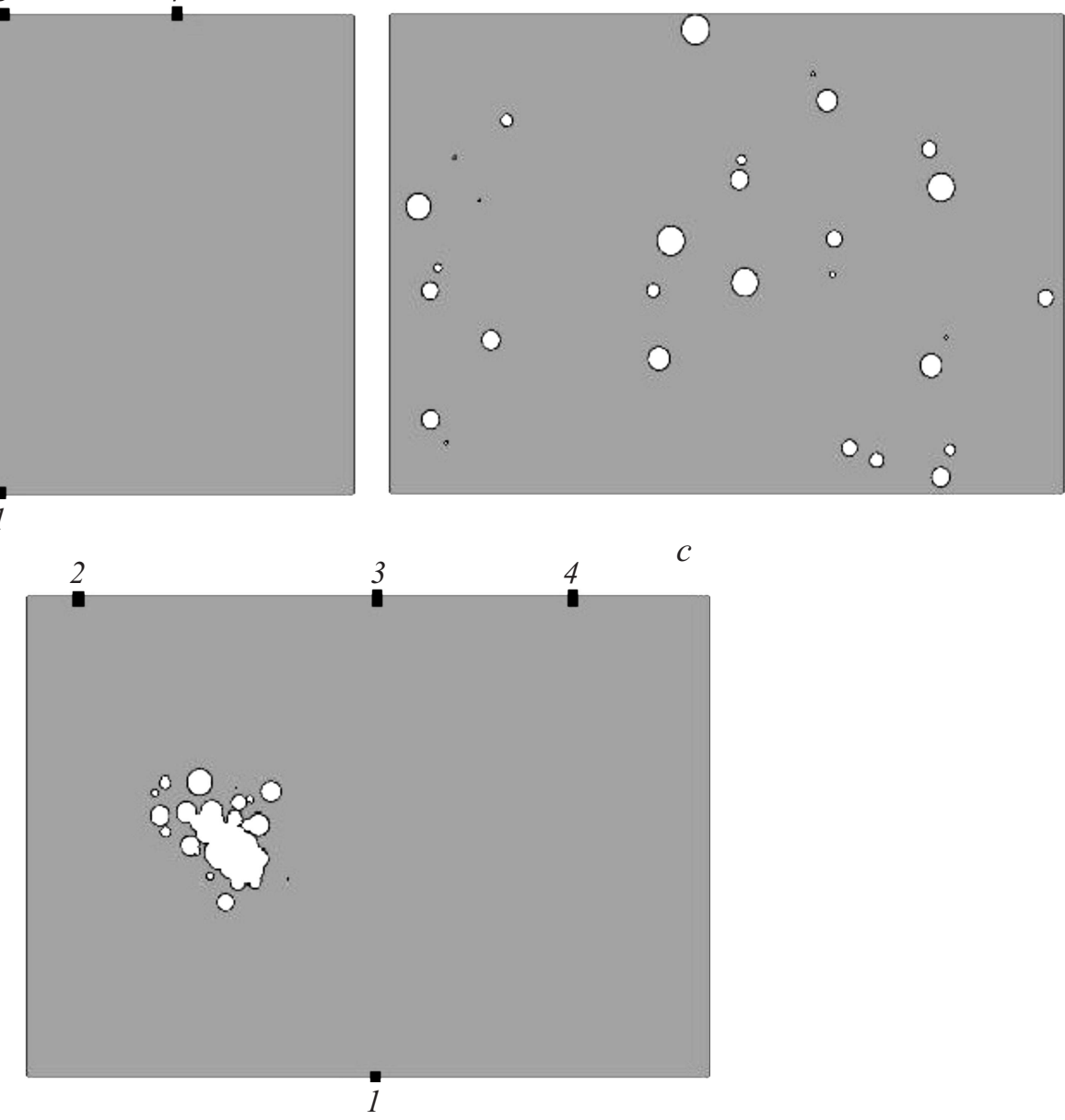
равномерным случайным распределением дефектов, $c$ ) - плита с нормальным распределением дефектов.

центров пор в пространстве - равномерное и нормальное со стандартными отклонениями 0.1 и $0.2 \mathrm{~m}$. Равномерное распределение (рис. $1, b$ ) отвечает начальной делокализованной стадии процесса разрушения, часто наблюдающейся в гетерогенных материалах. Нормальное распределение моделирует рост очага разрушения (рис. 1,c). Отметим, что очаг помещался на линии от излучающего датчика 1 к приемному датчику 2 с целью изучить возможность выявления места образования очага рассматриваемым методом. Радиусы отверстий предполагались равномерно распределенными в диапазоне $0-0.05 \mathrm{~m}$.

Расчет смещений производился при разных степенях дефектности пластины методом конечных элементов. Шаг интегрирования по времени составлял $10^{-5} \mathrm{~s}$. При таком выборе временного шага и соответствующем пространственном разбиении полученные решения обладали хорошей сходимостью и были устойчивы во всем диапазоне интегрирования даже без учета затухания.

На основе полученных временных зависимостей нормальной компоненты вектора смещения рассчитывались кроссрекуррентные матрицы (2) для разных значений поврежденности, где в качестве векторов $\mathbf{X}$ и $\mathbf{Y}$ выступали $y$ компоненты смещений на датчиках 2-4. Данные для неповрежденного образца использовались как опорные. С целью количественного описания изменений $\mathrm{RP}$ при увеличении поврежденности материала применялся количественный рекуррентный анализ (RQA). Для построения RP и расчета RQA использовался свободно распространяемый пакет программ CRP Toolbox [3].

B качестве примера кроссрекуррентные графики смещений на датчике 3 для однородного распределения пор в пространстве приведены на рис. 2 для разного содержания пор в образце (до 11\% дефектности) [5]. Во всех случаях проявляется четкая структурированность графика, свидетельствующая о детерминированности процесса. В тоже время график на рис. 2, $a$ (в отличие от рис. $2, b, c)$ симметричен относительно главной диагонали, проходящей из левого нижнего угла в правый верхний. Это тривиальный результат, поскольку для рекуррентного графика бездефектного образца на рис. 2, $a$ пары точек $i, j$ и $j, i$ эквивалентны. В то же время главная диагональ на рис. $2, b$ сильно деформирована, a на рис. 2,c практически отсутствует, что говорит 

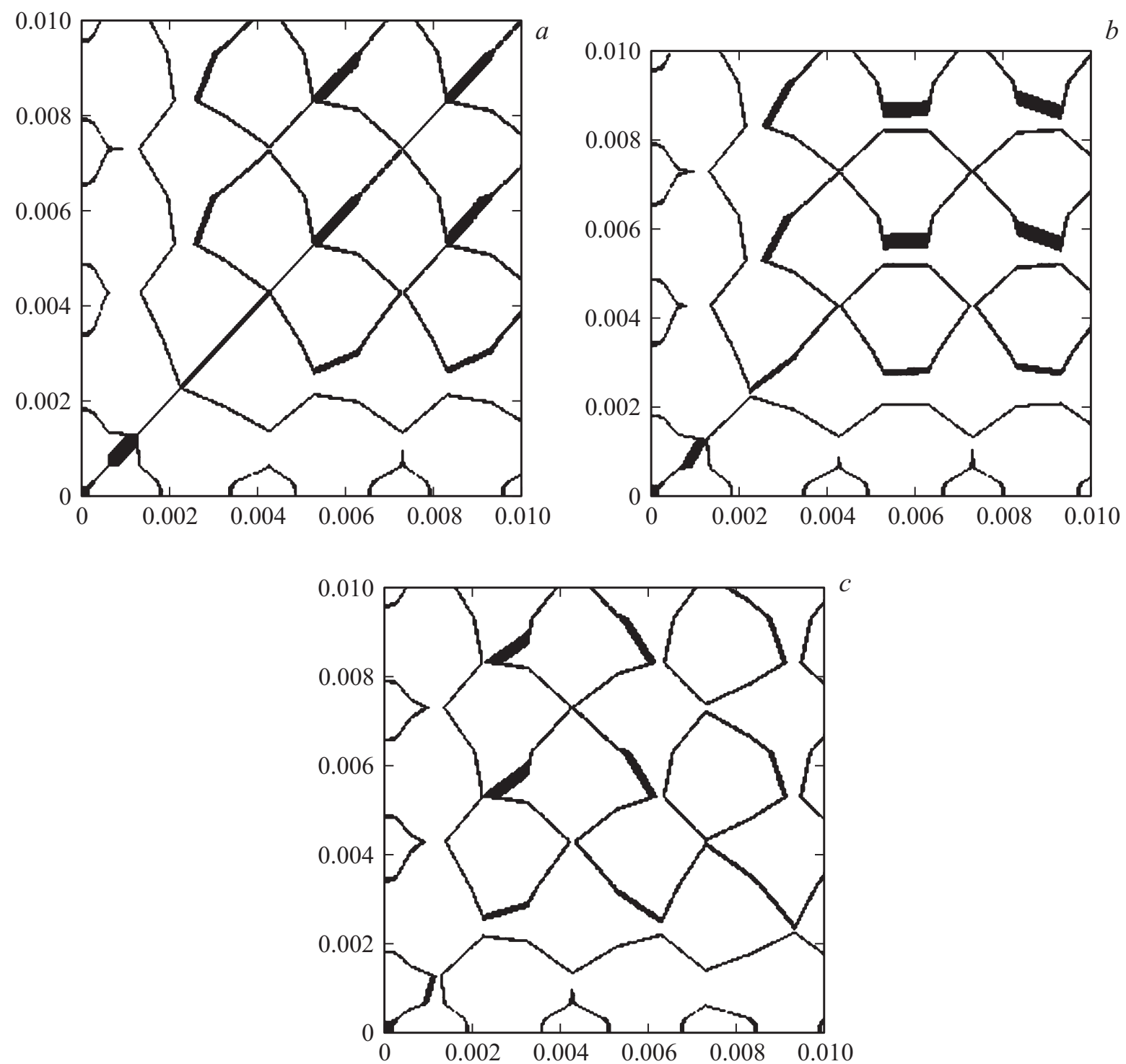

Рис. 2. Рекуррентные графики для бездефектного образца и двух дефектных $(D \approx 2 \%$ и $D \approx 11 \%)$.

о влиянии степени дефектности на кроссрекуррентные графики. Начальные участки графиков отличаются от остальной их части, что связано с нестационарностью процесса, так как через $10^{-3} \mathrm{~s}$ после начала отсчета времени действие силового импульса прекращается.

Итогом применения к рекуррентным графикам количественного рекуррентного анализа (RQA) явились 17 параметров (таких как уровень возврата, детерминизм, максимальные и средние длины диагональных и вертикальных линий и т.д. $[3,6])$. Для выявления характера зависимости большого числа параметров от степени дефектности образца целесообразно использовать метод главных компонент. В качестве переменных использовались параметры RQA, а в качестве образцов плиты с различным числом отверстий (бездефектная плита отсутствует) и различного характера дефектности (однородным или нормальным распределением с разной дисперсией центров отверстий в пространстве).
На рис. 3, $a$ изображен график счетов для плиты с однородной дефектностью, а на рис. $3, b$ для развивающегося очага разрушения со стандартным отклонением $0.1 \mathrm{~m}$, отвечающие изображениям на рис. $1, b$ и $c$. Графики построены в осях главная компонента 1 (ГК1)главная компонента 2 (ГК2). Номера точек 2-10 (по увеличению поврежденности) соответствуют датчику 2, номера $12-20$ - датчику 3 , а номера $22-30-$ датчику 4. На обоих рисунках видно, что первая главная компонента соответствует увеличению дефектности, а из pис. $1, c$ следует, что вторая главная компонента характеризует положение приемного датчика. По мере развития разрушения точки на графике счетов кластеризуются, что отвечает кластеризации разрушения в реальном пространстве. Поэтому характер кластеризации точек на графике счетов может служить прогностическим признаком процесса разрушения. На начальных стадиях процесса (до начала кластеризации) отчетливо видна 

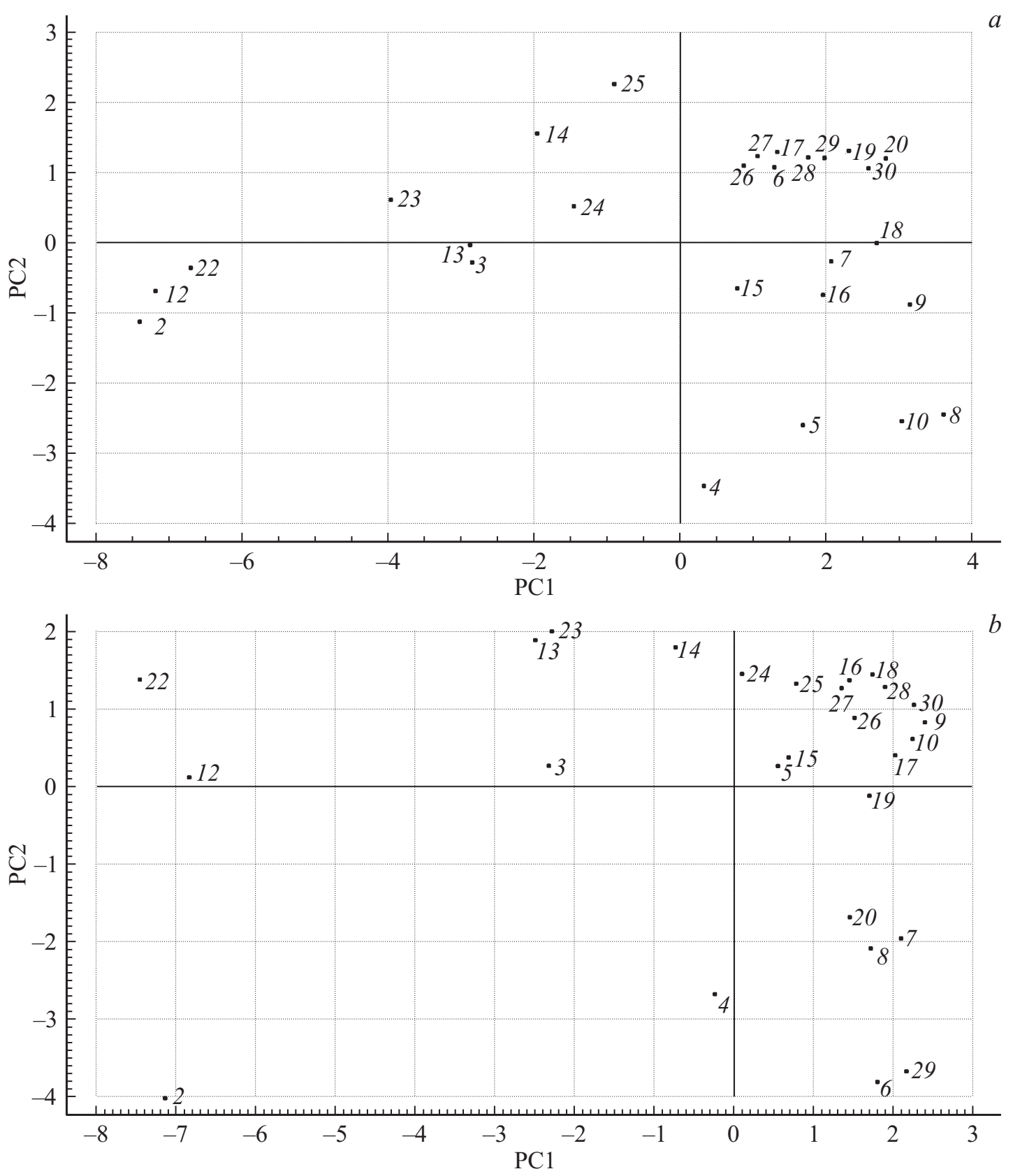

Рис. 3. Графики счетов для плиты с однородным $(a)$ и нормальным (стандартное отклонение $0.1 \mathrm{~m})(b)$ распределением дефектов в пространстве.

разница делокализованного (однородного) и локализованного процессов. Точки, отвечающие датчику 2 на рис. $3, b$ всегда лежат ниже точек, соответствующих датчикам 3 и 4, поскольку очаг растет на направлении от датчика 1 к датчику 2. В то же время для делокализованного процесса точки, соответствующие разным датчикам, лежат значительно ближе друг к другу (фактически их отличие в бездефектном образце отражает ассиметричное расположение датчиков). Таким образом, при помощи главной компоненты 2 рассматриваемый метод позволяет определить направление на очаг разрушения.
На рис. 4 приведены графики нагрузок для образцов с различной дефектностью и различным характером пространственного накопления дефектов. Рис. 4, $a$ отвечает однородному накоплению дефектов в пространстве (соответствует рис. $1, b$ ), рис. $4, b$ отвечает гауссовскому очагу со стандартным отклонением $0.2 \mathrm{~m}$, а рис. $4, c-$ гауссовскому очагу со стандартным отклонением $0.1 \mathrm{~m}$. Увеличение компактности очага (от $a$ до $c$ ) приводит к кластеризации точек вблизи левой части оси ГК1 (горизонтальная ось). Поэтому вид этого кластера свидетельствует о степени опасности растущего очага разрушения. Кластеризация точек на графике нагрузок 

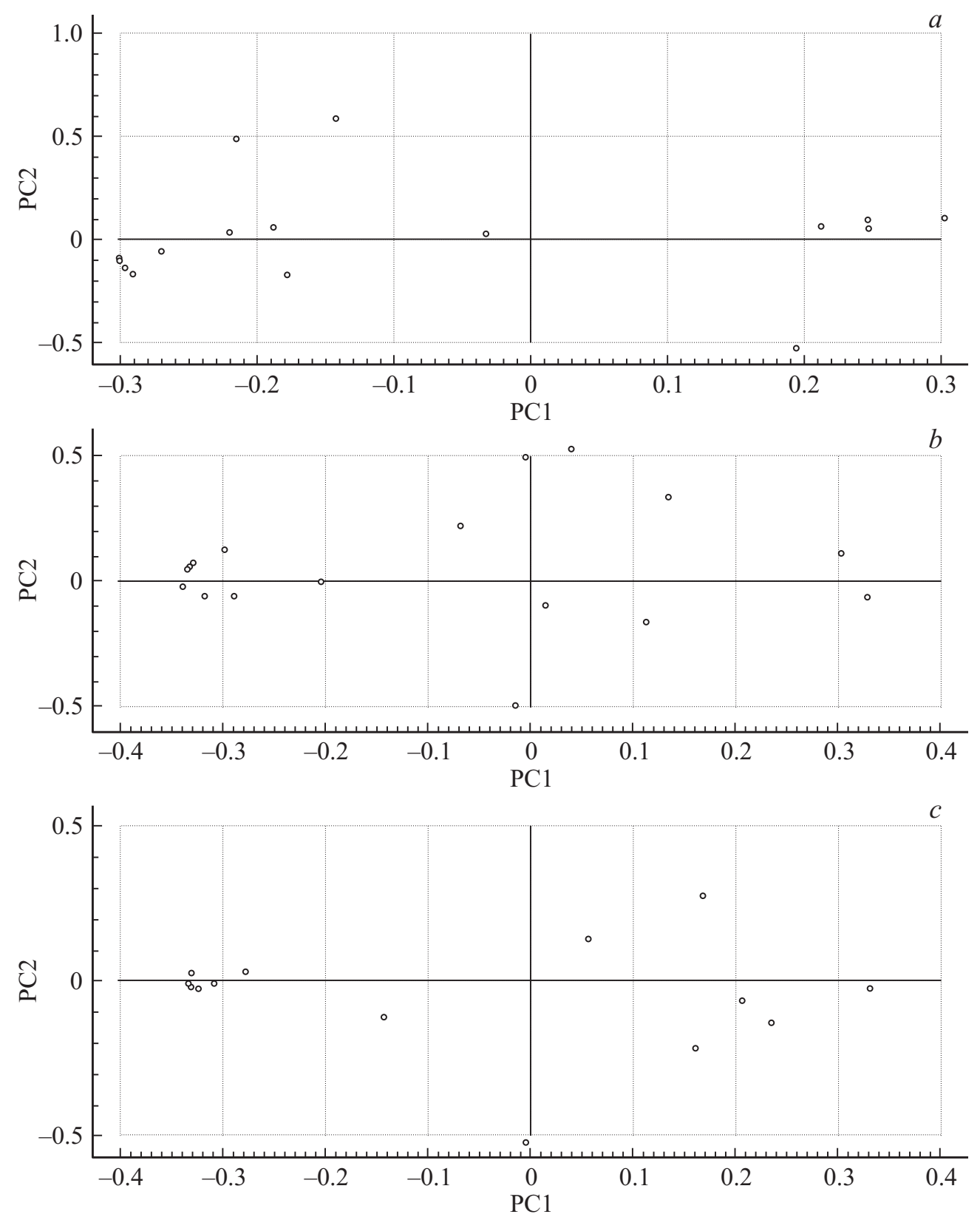

Рис. 4. Графики нагрузок для плиты с однородным (a), нормальным (стандартное отклонение $0.2 \mathrm{~m})(b)$ и нормальным со стандартным отклонением $0.1 \mathrm{~m}(c)$ распределением дефектов в пространстве.

свидетельствует об увеличении корреляции между соответствующими переменными, среди которых в основном содержатся параметры, связанные с диагональными линиями (детерминизм DET, средняя длина диагональных линий Lmean, энтропия диагональных линий EntrL).

Подводя итоги, можно сказать, что метод рекуррентных графиков представляется перспективным в применении к звуковой и ультразвуковой дефектоскопии. На рассмотренных примерах показано, что он позволяет различать делокализованный и локализованный характер разрушения, а также выявлять направление на очаг разрушения. При этом метод применим для нестационарных процессов в нелинейных системах.

\section{Список литературы}

[1] J.-P. Eckmann, S.O. Kampost, D. Ruelle. Europhys. Lett. 4, 973 (1987)

[2] Н.В. Золотова, Д.И. Понявин. Вопросы геофизики. Уч. записки № 438: СПБГУ 38, 203 (2005).

[3] N. Marwan, M.C. Romano, M. Thiel, J. Kurths. Phys. Rep. 438, 237 (2007).

[4] M. Nichols, S.T. Trickey, M. Seaver. Mechan. Syst. Signal Proc. 20, 421 (2006).

[5] В.Л. Гиляров. ФТТ 59, 1765 (2017).

[6] N. Marwan, J. Donges, Y. Zou, R. Donner, J. Kurths. Phys. Lett. A 373, 4246 (2009). 